\title{
Prediction and observation of intermodulation sidebands from anharmonic phonons in $\mathrm{NaBr}$
}

\author{
Y. Shen, ${ }^{1}$ C. N. Saunders $\odot,{ }^{1}$ C. M. Bernal $\odot,{ }^{1}$ D. L. Abernathy $\odot,{ }^{2}$ T. J. Williams, ${ }^{2}$ M. E. Manley $\odot,{ }^{3}$ and B. Fultz $\oplus^{1, *}$ \\ ${ }^{1}$ Department of Applied Physics and Materials Science, California Institute of Technology, Pasadena, California 91125, USA \\ ${ }^{2}$ Neutron Scattering Division, Oak Ridge National Laboratory, Oak Ridge, Tennessee 37831, USA \\ ${ }^{3}$ Materials Science and Technology Division, Oak Ridge National Laboratory, Oak Ridge, Tennessee 37831, USA
}

(Received 14 November 2020; accepted 16 March 2021; published 12 April 2021)

\begin{abstract}
A quantum Langevin model, like models from optomechanics, was developed for phonons. It predicts intermodulation phonon sidebands (IPSs) in anharmonic crystals. Ab initio calculations of anharmonic phonons in rock-salt $\mathrm{NaBr}$ showed these spectral features as many-body effects. Modern inelastic neutron scattering measurements on a crystal of $\mathrm{NaBr}$ at $300 \mathrm{~K}$ revealed diffuse intensity at high phonon energy from a predicted upper IPS. The transverse optical (TO) part of the new features originates from phonon intermodulation between the transverse acoustic (TA) and TO phonons. The longitudinal optical spectral features originate from three-phonon coupling between the TA modes and the TO lattice modes. The partner lower IPS proves to be an intrinsic localized mode. Interactions with the thermal bath broaden and redistribute the spectral weight of the IPS pair. These sidebands are a probe of the anharmonicity and quantum noise of phonons in $\mathrm{NaBr}$ and suggest novel interactions between photons and phonons.
\end{abstract}

DOI: 10.1103/PhysRevB.103.134302

\section{INTRODUCTION}

Phonons, quantized excitations of vibrational modes in crystals, bear resemblance to photons, quantized excitations of electromagnetic fields. Both obey the Planck statistics of bosons, and their quintessential models have similar Hamiltonians $\mathcal{H}_{0}=\hbar \omega\left(a^{\dagger} a+\frac{1}{2}\right)$, where $\hbar \omega$ is the energy of an individual phonon or photon, and $a^{\dagger} a$ gives the number of them. Phonons and photons exist in different media, so their properties are explained differently. For phonons, harmonic equations of motion are formulated as eigenvalue problems that give dispersions of frequency vs wave vector $\omega(\vec{k})[1,2]$. The degrees of freedom in three dimensions allow $3 \mathcal{R}$ dispersions, where $\mathcal{R}$ is the number of atoms in the translationally periodic unit cell. The harmonic model is readily extended to a quasiharmonic model to account for how frequencies shift with volume. Anharmonic models based on many-body perturbation theory [3-5] can account for how phonon frequencies shift with temperature alone and how finite phonon lifetimes originate with interactions between phonons. Perturbation theory couples the phonon modes, but the $3 \mathcal{R}$ dispersions are retained. To date, these $3 \mathcal{R}$ dispersions have been consistent with experimental observations. Exceptions are predictions [6,7] and experimental reports of intrinsic localized modes (ILMs) [8-12]. There are different viewpoints about the experimental evidence for ILMs, however [13-15].

In the field of laser-cavity physics, a quantized mechanical motion is coupled to photons in a cavity. When tuning the laser across the resonant frequency of the cavity, cooling or heating of the mechanical system generates sidebands about the main

*btf@caltech.edu resonance [16-23]. Photon-phonon couplings in laser-cavity experiments have similarities to anharmonic phonon-phonon couplings in crystals. The formal similarities motivate the question, "Do thermally driven asymmetric sidebands exist in the phonon spectra of anharmonic crystals?" To date, there has been no experimental evidence for this.

Advances in the sensitivity of methods for inelastic neutron scattering (INS) on single crystals motivated an examination of this question. After these experimental methods are described, this paper presents a quantum Langevin model for equilibrium phonon populations. INS data are presented on an anharmonic material, rock-salt $\mathrm{NaBr}$, revealing a diffuse spectral band at $300 \mathrm{~K}$. The diffuse band is predicted qualitatively by $a b$ initio calculations and perturbation theory with cubic perturbations to second order. This ab initio method with perturbation theory is used for identifying the specific phonon energies and branches involved in creating the diffuse band. The quantum Langevin model is not limited to small anharmonicity, however. It successfully explains the intensity and asymmetry of the intermodulation phonon sidebands (IPSs) through the anharmonic coupling of two phonons and their interactions with a thermal bath of other phonons in the crystal.

\section{EXPERIMENTAL MEASUREMENTS AND AB INITIO CALCULATIONS}

\section{A. INS experiments}

The measurements used a high-purity single crystal of $\mathrm{NaBr}$. Crystal quality was checked by $\mathrm{x}$-ray and neutron diffraction. The single crystal of [001] orientation was suspended in an aluminum holder, which was mounted in a closed-cycle helium refrigerator for the $10 \mathrm{~K}$ measurement 
and in a low-background electrical resistance vacuum furnace for measurements at $300 \mathrm{~K}$.

The INS data were acquired with the time-of-flight Wide Angular-Range Chopper Spectrometer (ARCS) [24] at the Spallation Neutron Source at Oak Ridge National Laboratory, using neutrons with an incident energy $E_{i}$ of $50 \mathrm{meV}$. The techniques and material were similar to those reported previously [25], but the previous study had a problematic background at the precise energy transfers of interest here [26]. We therefore acquired an entirely new dataset with $E_{i}=$ $50 \mathrm{meV}$.

For each measurement, time-of-flight neutron data were collected from 201 rotations of the crystal in increments of $0.5^{\circ}$ about the vertical axis. Data reduction gave the fourdimensional (4D) scattering function $S(\mathbf{Q}, \varepsilon)$, where $\mathbf{Q}$ is the three-dimensional (3D) wave vector of momentum transfer, and $\varepsilon$ is the phonon energy (from the neutron energy loss). Measurements were performed to evaluate the background from an empty can. To correct for nonlinearities of the ARCS instrument, offsets of the $q$ grid were corrected to the first order by fitting a set of 76 in situ Bragg diffractions, which were transformed to their theoretical positions in the reciprocal space of the $\mathrm{NaBr}$ structure. The linear transformation matrix had only a small deviation $(<0.02)$ from the identity matrix, showing that the original data had good quality and that linear corrections for $q$ offsets were adequate. After subtracting the empty-can background and removing multiphonon scattering with the incoherent approximation, the higher Brillouin zones were folded back [25,27] into an irreducible wedge in the first Brillouin zone to obtain the spectral intensities. Further information about the ARCS background is given in the Supplemental Material [28].

The temperature dependence of the low-energy dynamics of $\mathrm{NaBr}$ was measured with higher resolution with the HB3 triple axis spectrometer at the High Flux Isotope Reactor (HFIR) of Oak Ridge National Laboratory. Pyrolytic graphite PG(002) was used for both the monochromator and the analyzer. The spectrometer was operated with a filtered, fixed final neutron energy of $14.7 \mathrm{meV}$ with horizontal collimation 48:40:40:120. The $\mathrm{NaBr}$ crystal was mounted in a vacuum furnace with the (HHL) reflections in the scattering plane. Measurements were made in transverse geometry near (113) along $\vec{Q}=[H, H, 3]$ at temperatures from 300 to $723 \mathrm{~K}$.

\section{B. Ab initio calculations}

All density functional theory (DFT) calculations were performed with the Vienna $A b$ initio Simulation Package (VASP) package using a plane-wave basis set [29-32] with projector augmented wave (PAW) pseudopotentials [33] and the Perdew-Burke-Ernzerhof (PBE) exchange correlation functional [34]. The Born effective charges and dielectric constants were obtained by DFT calculations in VASP [35]. A correction for the nonanalytical term of the long-ranged electrostatics was performed in both quasiharmonic and anharmonic calculations [36]. All calculations used a kineticenergy cutoff of $550 \mathrm{eV}$, a $5 \times 5 \times 5$ supercell of 250 atoms, and a $3 \times 3 \times 3 k$-point grid. The phonon self-energy was calculated with a $35 \times 35 \times 35 q$ grid. Calculations of phonons in the quasiharmonic approximation (QHA) used PHONOPY
[37]. The QHA method allows the frequencies and entropy of phonons to vary with volume and thermal occupancy factor. It does not include thermal displacements of individual atoms off periodic sites, as arise in molecular dynamics, for example, and the QHA was not accurate for predicting the thermal expansion of $\mathrm{NaBr}$ [25].

The stochastically initialized temperature dependent effective potential (sTDEP) [38-40] method was used to accelerate the traditional ab initio molecular dynamics (AIMD) and calculate anharmonic phonon dispersions at finite temperatures. The method for $\mathrm{NaBr}$ was described previously [25]. In short, the phonon frequencies were obtained from the dynamical matrix for the quadratic force constants and then corrected by the real $(\Delta)$ and imaginary $(\mathrm{i} \Gamma)$ parts of the phonon selfenergy from many-body theory $[4,5]$. The imaginary part, which gives phonon lifetime broadening, was calculated with the third-order force constants

$$
\begin{aligned}
\Gamma_{\lambda}(\Omega)= & \frac{\hbar \pi}{16} \sum_{\lambda^{\prime} \lambda^{\prime \prime}}\left|\Phi_{\lambda \lambda^{\prime} \lambda^{\prime \prime}}\right|^{2}\left\{\left(n_{\lambda^{\prime}}+n_{\lambda^{\prime \prime}}+1\right)\right. \\
& \times \delta\left(\Omega-\omega_{\lambda^{\prime}}-\omega_{\lambda^{\prime \prime}}\right)+\left(n_{\lambda^{\prime}}-n_{\lambda^{\prime \prime}}\right) \\
& \left.\times\left[\delta\left(\Omega-\omega_{\lambda^{\prime}}+\omega_{\lambda^{\prime \prime}}\right)-\delta\left(\Omega+\omega_{\lambda^{\prime}}-\omega_{\lambda^{\prime \prime}}\right)\right]\right\},
\end{aligned}
$$

where $\Omega=E / \hbar$ is the probing energy. The real part was obtained by a Kramers-Kronig transformation

$$
\Delta(\Omega)=\mathcal{P} \int \frac{1}{\pi} \frac{\Gamma(\omega)}{\omega-\Omega} d \omega .
$$

Equation (1) is a sum over all possible three-phonon interactions, where $\Phi_{\lambda \lambda^{\prime} \lambda^{\prime \prime}}$ is the three-phonon matrix element obtained from the cubic force constants by Fourier transformation, $n$ is the Bose-Einstein thermal occupation factor giving the number of phonons in each mode, and the $\delta$ functions conserve energy and momentum. Details were given in the supplemental materials in our previous work [25].

\section{QUANTUM LANGEVIN MODEL}

We start with the Hamiltonian of three coupled phonons denoted as $j, j^{\prime}$, and $j^{\prime \prime}$ :

$$
\mathcal{H}_{\mathrm{sys}}=\mathcal{H}_{0}+\hbar \eta\left(\hat{a}_{j}^{\dagger}+\hat{a}_{j}\right)\left(\hat{a}_{j^{\prime}}^{\dagger}+\hat{a}_{j^{\prime}}\right)\left(\hat{a}_{j^{\prime \prime}}^{\dagger}+\hat{a}_{j^{\prime \prime}}\right),
$$

where $\mathcal{H}_{0}=\sum_{k=j, j^{\prime}, j^{\prime \prime}} \hbar \omega_{k}\left(\hat{a}_{k}^{\dagger} \hat{a}_{k}+\frac{1}{2}\right)$ is the Hamiltonian for three uncoupled, independent oscillators, and $\eta$ parameterizes the coupling strength. However, there is also a special case where only two types of phonons are involved in this interaction process. Taking $j^{\prime}=j^{\prime \prime}$ as an example, the system Hamiltonian is then

$$
\mathcal{H}_{\text {sys }}=\mathcal{H}_{0}+\hbar \frac{\eta}{2}\left(\hat{a}^{\dagger}+\hat{a}\right)^{2}\left(\hat{b}^{\dagger}+\hat{b}\right),
$$

where now $\hat{a}$ denotes the composite phonon mode with $j^{\prime}=$ $j^{\prime \prime}, \hat{b}$ denotes the $j$ mode, and the $\frac{1}{2}$ is added for later convenience. Confining our interest to terms under the rotating wave approximation (RWA) in quantum optics, we eliminate the terms $a a b^{\dagger}$ and $a^{\dagger} a^{\dagger} b$ (and $a a b$ and $a^{\dagger} a^{\dagger} b^{\dagger}$ that do not conserve energy):

$$
\mathcal{H}_{\mathrm{sys}}=\mathcal{H}_{0}+\hbar \frac{\eta}{2}\left(\hat{a}^{\dagger} \hat{a}+\hat{a} \hat{a}^{\dagger}\right)\left(\hat{b}^{\dagger}+\hat{b}\right) .
$$


(a)
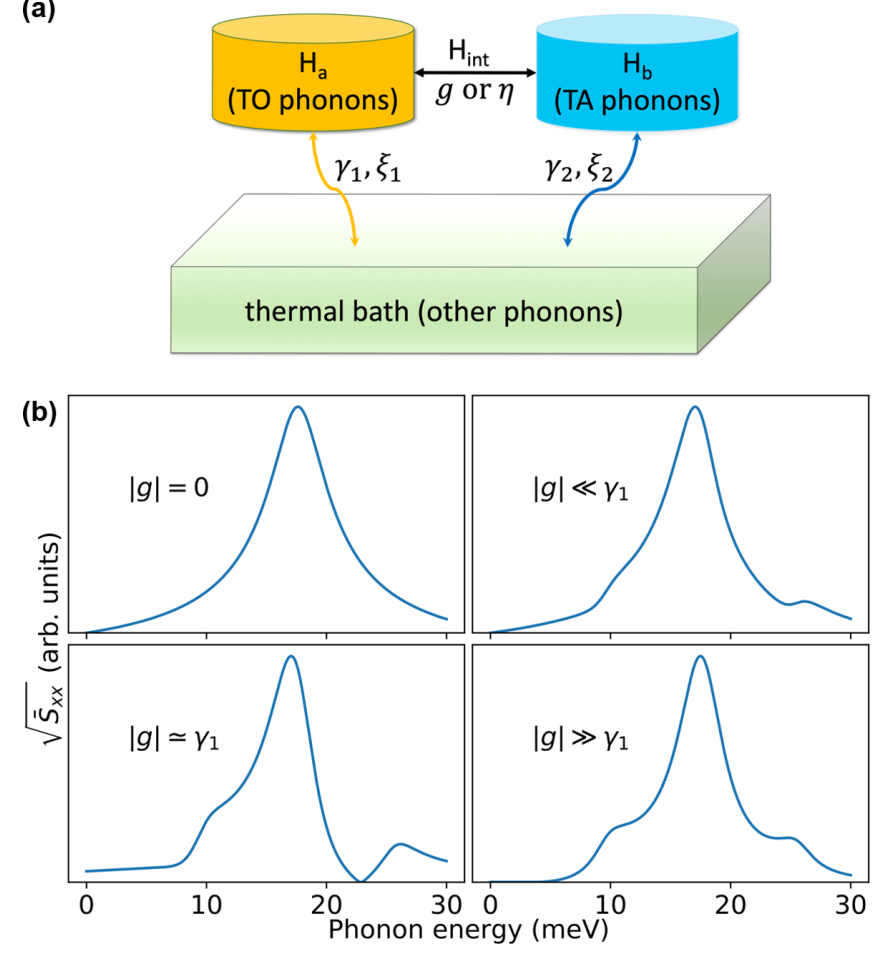

FIG. 1. Phonon self-transduction block diagram and its features. (a) The transverse optical (TO) phonons and the transverse acoustic (TA) phonons within 7-9 meV are coupled by phonon-phonon interactions. Meanwhile, they are in thermal equilibrium with the bath, which is an ensemble of other phonons. (b) Power spectral density for coupling strength $|g|$ from none, weak, intermediate, strong.

The general method of input-output theory [16,41] gives the Heisenberg-Langevin equations of motion for the two modes:

$$
\begin{gathered}
\dot{\hat{a}}=-i \omega_{1} \hat{a}-i \eta \hat{a}\left(\hat{b}^{\dagger}+\hat{b}\right)-\frac{\gamma_{1}}{2} \hat{a}-\sqrt{\gamma_{1}} \hat{\xi}_{1}, \\
\dot{\hat{b}}=-i \omega_{2} \hat{b}-i \frac{\eta}{2}\left(\hat{a}^{\dagger} \hat{a}+\hat{a} \hat{a}^{\dagger}\right)-\frac{\gamma_{2}}{2} \hat{b}-\sqrt{\gamma_{2}} \hat{\xi}_{2} .
\end{gathered}
$$

Here, $\gamma_{1}$ and $\gamma_{2}$ are decay rates of the two modes, giving phonon linewidths in energy. The other phonons are modeled as a thermal bath, described by stochastic operators $\xi_{1}(t)$ and $\xi_{2}(t)$. These satisfy the correlation conditions: $\left\langle\hat{\xi}^{\dagger}(t) \hat{\xi}\left(t^{\prime}\right)\right\rangle=n \delta\left(t-t^{\prime}\right)$ and $\left\langle\hat{\xi}(t) \hat{\xi}^{\dagger}\left(t^{\prime}\right)\right\rangle=(n+1) \delta\left(t-t^{\prime}\right)$, where $n$ is the equilibrium Planck thermal occupation factor $n=\left[\exp \left(\hbar \omega / k_{\mathrm{B}} T\right)-1\right]^{-1}$ (or the Bose-Einstein factor for zero chemical potential). These correlation conditions apply to both modes 1 and 2, a situation that differs from optomechanical systems where correlations of the stochastic variable $\xi_{1}$ for input noise of the optical photon do not scale with equilibrium thermal occupancies. Figure 1(a) depicts relationships between the transverse acoustic (TA) and transverse optical (TO) phonons and the thermal bath of other phonons, showing correspondences to the physical quantities of input-output theory.

Using the concept of intermodulation, a classical analysis by representing the phonon amplitudes of $\hat{a}$ as Fourier decomposition of sidebands [19] shows that $\hat{a}$ comprises the frequency components $\omega_{1}$ (first order), $\omega_{1} \pm \omega_{2}$ (second-order distortion), $\omega_{1} \pm 2 \omega_{2}$ (third-order distortion), etc. Secondorder effects are identified by transforming to a frame moving at the central frequency $\omega_{1}$ by replacing $\hat{a}(t) \rightarrow[\alpha+$ $\hat{c}(t)] e^{-i \omega_{1} t}$ and $\hat{\xi}_{1}(t) \rightarrow\left[\xi_{\text {in }}+\hat{\xi}_{1}(t)\right] e^{-i \omega_{1} t}$, where we take $\alpha$ to be real without loss of generality. This gives linearized equations of motion

$$
\begin{gathered}
\dot{\hat{c}}=-i g\left(\hat{b}^{\dagger}+\hat{b}\right)-\frac{\gamma_{1}}{2} \hat{c}-\sqrt{\gamma_{1}} \hat{\xi}_{1}, \\
\dot{\hat{b}}=-i \omega_{2} \hat{b}-i g\left(\hat{c}^{\dagger}+\hat{c}\right)-\frac{\gamma_{2}}{2} \hat{b}-\sqrt{\gamma_{2}} \hat{\xi}_{2},
\end{gathered}
$$

where $g=\eta \alpha$ is the coupling strength. A straightforward calculation (see Supplemental Material [28]) obtained the symmetrized power spectral density of displacement as

$$
\begin{aligned}
\bar{S}_{x x}[\omega]= & \frac{\hbar \gamma_{1}\left(n_{1}+\frac{1}{2}\right)}{2 m \omega_{1}}\left(\left|\chi_{a,-}+2 \mathrm{i} \omega_{2} g^{2} \chi_{a,-}^{2} \chi_{b,-} \bar{\chi}_{b,-}\right|^{2}\right. \\
& \left.+\left|\chi_{a,+}-2 \mathrm{i} \omega_{2} g^{2} \chi_{a,+}^{2} \chi_{b,+} \bar{\chi}_{b,+}\right|^{2}\right)
\end{aligned}
$$

where the response functions are defined as

$$
\begin{gathered}
\chi_{a, \pm}^{-1}=-i\left(\omega \pm \omega_{1}\right)+\frac{\gamma_{1}}{2}, \\
\chi_{b, \pm}^{-1}=-i\left(\omega \pm \omega_{1}-\omega_{2}\right)+\frac{\gamma_{2}}{2}, \\
\bar{\chi}_{b, \pm}^{-1}=-i\left(\omega \pm \omega_{1}+\omega_{2}\right)+\frac{\gamma_{2}}{2} .
\end{gathered}
$$

The first term in parentheses in Eq. (10)

$$
\bar{S}_{x x}^{(+)}[\omega]=\frac{\hbar \gamma_{1}\left(n_{1}+\frac{1}{2}\right)}{2 m \omega_{1}}\left|\chi_{a,-}+2 \mathrm{i} \omega_{2} g^{2} \chi_{a,-}^{2} \chi_{b,-} \bar{\chi}_{b,-}\right|^{2},
$$

contributes spectral weight primarily to the positive frequency region. The other term $\bar{S}_{x x}^{(-)}$contributes to the negative.

In the absence of phonon coupling, i.e., $g=0$, Eq. (14) reduces to the thermal noise spectrum of a damped harmonic oscillator

$$
\bar{S}_{x x}^{(+), \text {th }}[\omega]=\frac{\hbar \gamma_{1}\left(n_{1}+\frac{1}{2}\right)}{2 m \omega_{1}} \frac{1}{\left(\omega-\omega_{1}\right)^{2}+\left(\gamma_{1} / 2\right)^{2}} .
$$

Figure 1(b) shows this is a Lorentzian function centered at $\omega_{1}$. Three other cases are shown: weak coupling $\left(|g| \ll \gamma_{1}\right)$, medium coupling $\left(|g| \simeq \gamma_{1}\right)$, and strong coupling $\left(|g| \gg \gamma_{1}\right)$. To identify an IPS in a real material, the phonon-phonon interactions must be at least in the medium coupling regime. Recently, we identified $\mathrm{NaBr}$ with the rock-salt structure as a highly anharmonic solid system [25], so it seemed an appropriate candidate for finding phonon intermodulation phenomena.

\section{RESULTS}

A spectral feature labeled "G" (ghost) in Fig. 2(b) appears at $300 \mathrm{~K}$. It is flat over the Brillouin zone with an energy of 25-26 meV. This feature does not belong to any of the six phonon branches expected for the rock-salt structure (as in the white dotted lines in Figs. 2(c) and 2(d) from the QHA.

Figures 2(a) and 2(c) show that, at $10 \mathrm{~K}$, the quasiharmonic and anharmonic calculations agree well with each other and with the experimental phonon dispersions. At $300 \mathrm{~K}$, the quasiharmonic model predicts neither the phonon broadening 

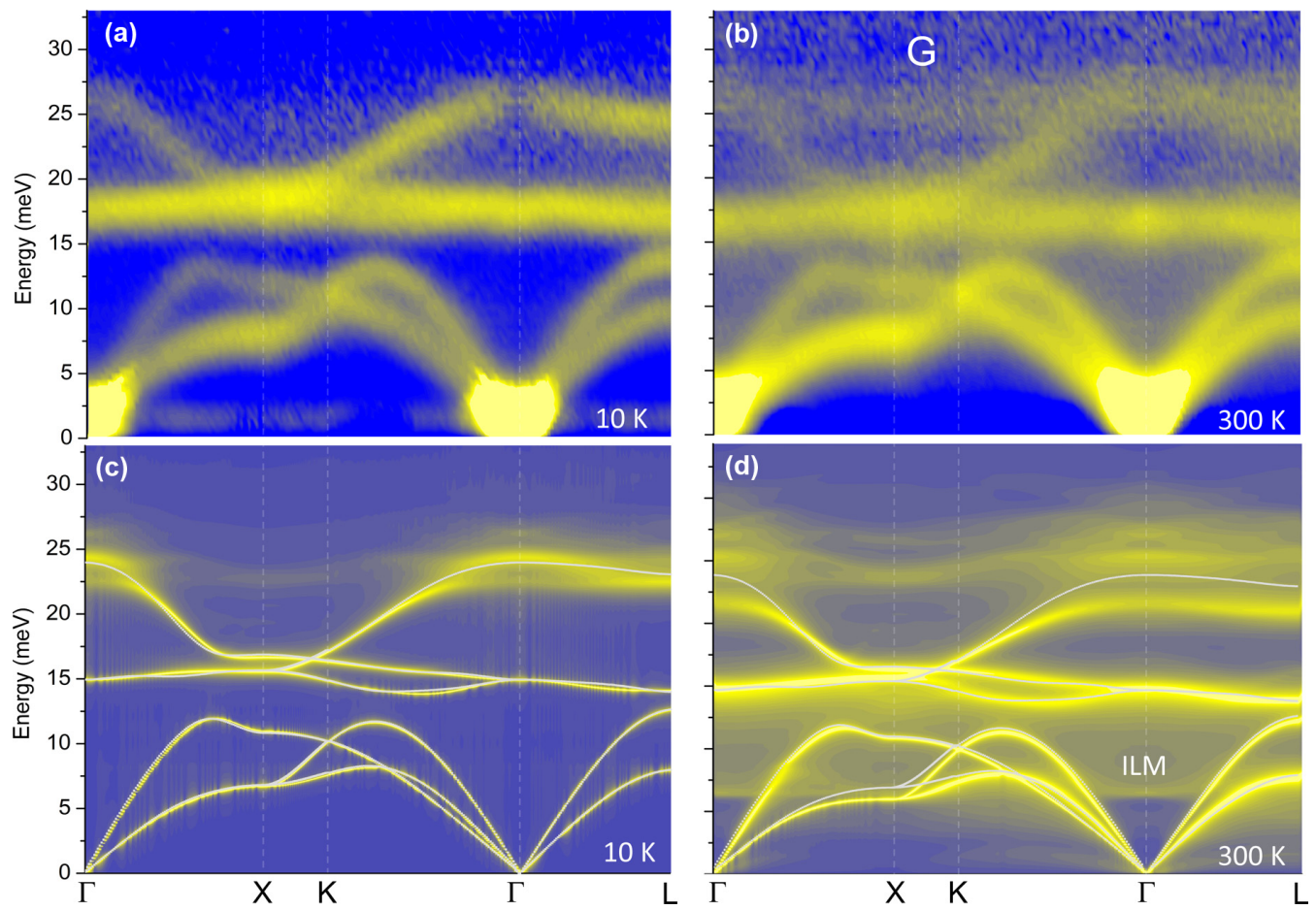

FIG. 2. Comparison between experimental and computational phonon dispersions of NaBr. (a) and (b) Two-dimensional (2D) slices through the four-dimensional scattering function $S(\mathbf{Q}, \varepsilon)$, where $\varepsilon=\hbar \omega$, along high symmetry lines in the first Brillouin zone. (a) and (b) are linear plots, with intensities corrected for thermal populations. (c) and (d) Phonons in $\mathrm{NaBr}$ calculated with the quasiharmonic approximation (thin white lines) and the full phonon spectral function with phonon self-energy corrections. Temperatures are labeled. The intermodulation phonon sideband (IPS) "G" is seen in the experimental and computational results around the $X$ point at $300 \mathrm{~K}$. The calculation also shows an ILM near the $\Gamma$ point at $300 \mathrm{~K}$. (c) and (d) are logarithmic plots of spectral weights.

nor most of the thermal energy shift. The anharmonic calculations, however, reproduce these features and further predict the ghost intensity around $25 \mathrm{meV}$, in good agreement with experiment. Figure 3 shows that the ghost disappears when the calculation neglects the three-phonon anharmonic interactions of $\mathrm{TA}+\mathrm{TO} \rightleftharpoons \mathrm{TO} /$ longitudinal optical (LO). Moreover, the
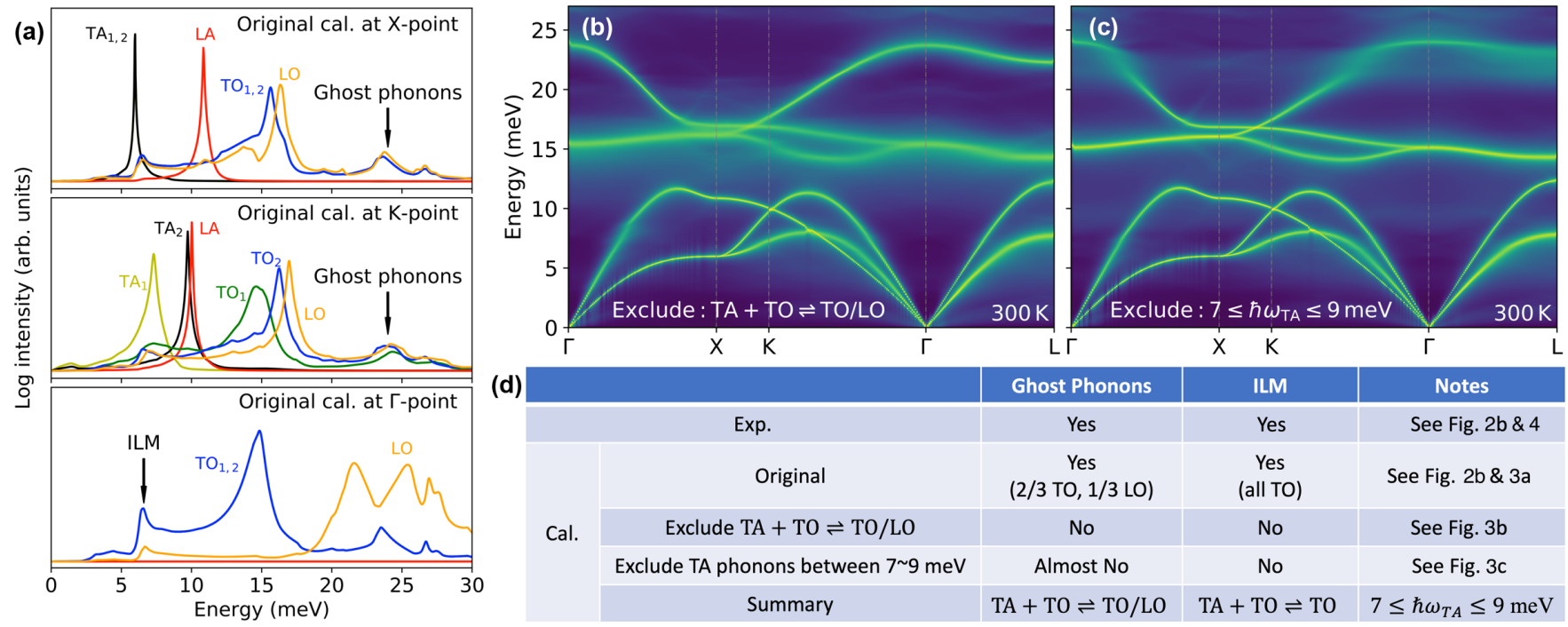

FIG. 3. Three-phonon processes associated with the intermodulation phonon sideband (IPS) and intrinsic localized mode (ILM). (a) Calculated phonon lineshapes at the high symmetry points of $X, K$, and $\Gamma$. The first two were used to identify the components of the IPS, and the calculated ILM is shown in the bottom panel. The phonon spectral function was recalculated (b) without the three-phonon processes of transverse acoustic (TA) + transverse optical (TO) $\rightleftharpoons \mathrm{TO} /$ longitudinal optical (LO), and (c) without TA phonons between 7-9 meV included in the three-phonon processes compared with the main result in Fig. 2(d). (d) Table of phonon processes for IPS and the ILM. 


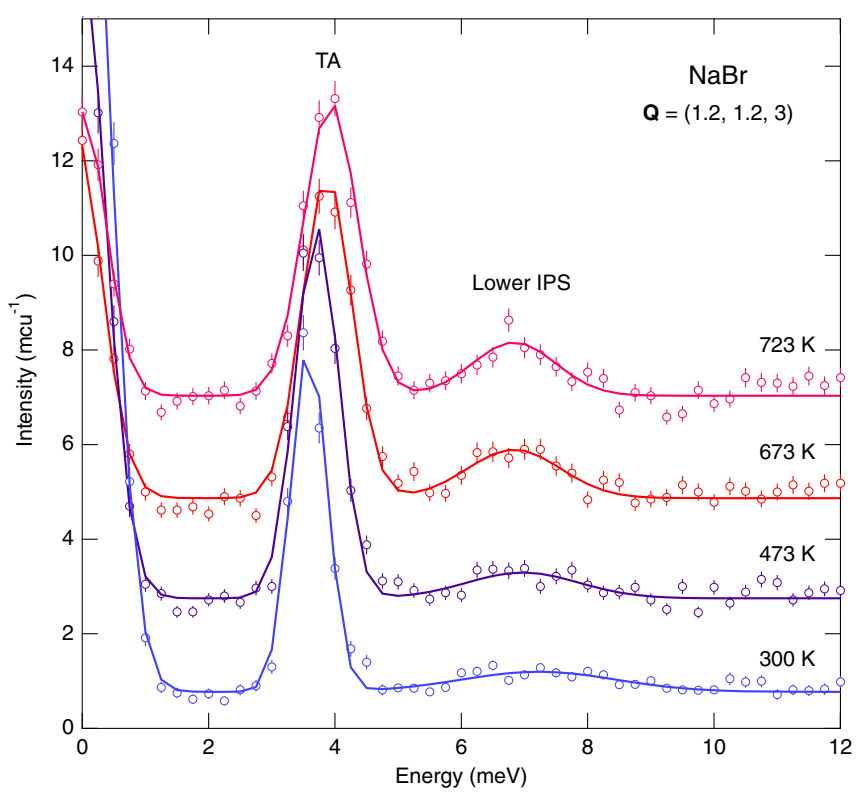

FIG. 4. Triple-axis energy scan, showing the temperature dependence of the spectral intensity distribution for the transverse acoustic (TA) and lower intermodulation phonon sideband (IPS) phonons at $\vec{Q}=(1.2,1.2,3)$.

participating TA phonons were shown to have energies between 7 and $9 \mathrm{meV}$. The diffuse features nearly vanish at $10 \mathrm{~K}$. (They should not vanish entirely, however, owing to effects of the zero-point occupancies of the TA and TO modes [27,42].) Finally, the computations showed that the features comprise optical modes, with polarizations distributed evenly over all transverse (two) and longitudinal (one) possibilities.

Similarly, the calculated ILM near the $\Gamma$ point is produced by $\mathrm{TA}+\mathrm{TO} \rightleftharpoons \mathrm{TO}$ in the calculation. Although the ILM is not definitive in Fig. 2(b) at $300 \mathrm{~K}$, it is well resolved at higher temperatures in the HB3 data of Fig. 4. This figure shows the temperature dependence of the lower IPS (i.e, the ILM) as observed in the (113) Brillouin zone at (1.2, 1.2, 3.0), along with the TA phonon. The spectral weight of this sideband gradually sharpens and intensifies with increasing temperature, and it shifts slightly to lower energy. The TA mode has an apparent stiffening with temperature, but this is an artifact from thermal expansion [43]. The TA mode also broadens with increasing temperature, as expected from the stronger coupling strength with increasing temperature, discussed below. Finally, the longitudinal acoustic (LA) mode is suppressed in the spectra of Fig. 4 because $\vec{Q}$ is nearly perpendicular to the polarization vector $\vec{e}$ of the LA mode, i.e., the direction of atom displacements in the mode. (If the LA mode was visible, its temperature dependence would follow approximately the TA mode.)

The three phonon modes in Eq. (1) are eigenstates of a dynamical matrix. Small anharmonic shifts and broadenings of these eigenstates do not produce new phonon branches or spectral features. Our anharmonic calculations obtained the diffuse features after applying a Kramers-Kronig transformation of Eq. (2) to the imaginary part of the phonon self energy of Eq. (1). The semiquantitative success is interesting because the calculations also predict a weak ILM $[12,44]$. The dispersions in Figs. 2(c) and 2(d) are on a logarithmic scale, however, and the ILM and ghost modes are much weaker in the calculation with perturbation theory than in the experimental intensities, discussed with Fig. 5.

Compared with Eq. (1), the Heisenberg-Langevin Eqs. (6) and (7) have no implicit assumption that the anharmonic perturbation is small. The intensities of the measured ghost modes are seen in Fig. 5, which are energy cuts at different $Q$ along high-symmetry directions through the experimental data of Fig. 2(b). The points near $X$ or $K$ [see Figs. 5(c)-5(g)] show an extra peak above $20 \mathrm{meV}$, which is distinct from the highest normal LO phonon branch.

Phonon centroids were obtained by fitting with the Levenberg-Marquardt nonlinear least square method for multiple Lorentzian functions, giving the fitting parameters listed in Table S1 in the Supplemental Material [28]. The fitting results were used to obtain average energies and linewidths of TO and TA phonons in the energy range of 7-9 meV (i.e., $\omega_{1}, \omega_{2}, \gamma_{1}$, and $\gamma_{2}$ ) for calculating sidebands with the Heisenberg-Langevin model. By performing averages over TA and TO peaks that were not impaired by overlaps with other peaks, $\omega_{1}=16.97(10), \omega_{2}=8.2(2), \gamma_{1}=3.6(10)$, and $\gamma_{2}=3.7(7) \mathrm{meV}$, where subscripts 1 and 2 denote TO and TA, respectively The average frequency of the ghost mode $\left(\omega_{\mathrm{G}}\right)$ was obtained from Figs. 5(c) $-5(\mathrm{~g})$ as $\omega_{\mathrm{G}}=25.9(5) \mathrm{meV}$, satisfying $\omega_{1}+\omega_{2} \simeq \omega_{\mathrm{G}}$.

The coupling strength was calculated by comparing the power intensity between the Heisenberg-Langevin model and the measured peak intensities $I_{\exp }(\omega)$ at $300 \mathrm{~K}$. Their ratio avoids scaling factors

$$
\frac{\bar{S}_{x x}^{(+)}\left[\omega_{1}\right]}{\bar{S}_{x x}^{(+)}\left[\omega_{1}+\omega_{2}\right]}=\frac{I_{\exp }\left(\omega_{1}\right) n\left(\omega_{1}\right)}{I_{\exp }\left(\omega_{1}+\omega_{2}\right) n\left(\omega_{1}+\omega_{2}\right) 2 / 3} .
$$

The terms in the left-hand side are derived in the Supplemental Material [28]. Here, $n(\omega)=\left[\exp \left(\hbar \omega / k_{\mathrm{B}} T\right)-1\right]^{-1}$ is the Planck distribution function [the thermal weight was corrected for the measured intensity shown in Figs. 2(a) and 2(b)], and the $\frac{2}{3}$ factor is included because two-thirds of the IPS feature are $\mathrm{G}-\mathrm{TO}$ phonons.

\section{DISCUSSION}

At room temperature, our analysis showed that the lower IPS should not be visible as a distinct peak. Also, the ARCS spectrometer has lower energy resolution at the energy of the lower sideband than at the energy of the upper sideband. The lower sideband is better seen with the HB3 instrument at higher temperatures (Fig. 4).

The $\mathbf{q}$ dependence of phonons in solids is not considered in Eqs. (6) and (7), and the conservation of crystal momentum is an added complexity that is not needed for other coupled quantum systems [16-23]. Figure 2 shows that, in $\mathrm{NaBr}$, however, the TO phonon branch and the upper IPS are largely flat and dispersionless. The TA phonons involved in the threephonon processes are in a small energy range of 7-9 meV, as shown above with Fig. 3. The phonon dispersions (Fig. 2) show that most of the TA phonons are in this energy range, forming plateaus reaching to the Brillouin zone boundary. Most TA phonons can be described with an average energy 

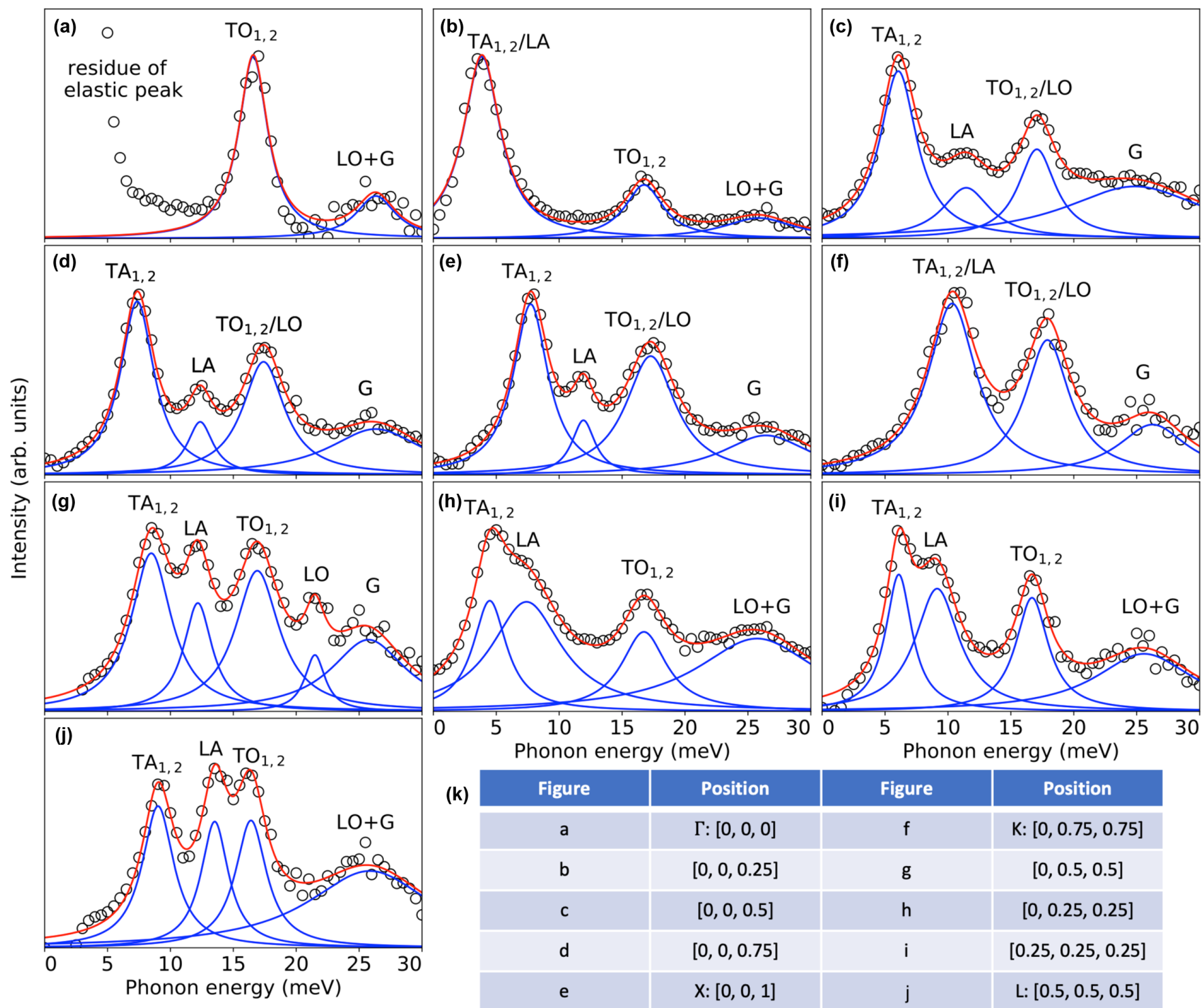

(k)

\begin{tabular}{|c|c|}
\hline Figure & Position \\
\hline a & $\Gamma:[0,0,0]$ \\
\hline$b$ & {$[0,0,0.25]$} \\
\hline c & {$[0,0,0.5]$} \\
\hline$d$ & {$[0,0,0.75]$} \\
\hline e & $X:[0,0,1]$ \\
\hline
\end{tabular}

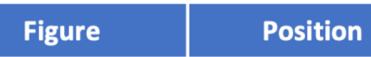

\begin{tabular}{|c|c|}
\hline$f$ & $K:[0,0.75,0.75]$ \\
\hline g & {$[0,0.5,0.5]$} \\
\hline h & {$[0,0.25,0.25]$} \\
\hline i & {$[0.25,0.25,0.25]$} \\
\hline j & L: $[0.5,0.5,0.5]$ \\
\hline
\end{tabular}

FIG. 5. Energy cuts at constant $\mathbf{q}$ through experimental dispersions of Fig. 2(b). (a)-(j) Experimental data are points; fitted peaks are in blue, and the cumulative fitting results are in red. (k) Table of the q-points for each panel.

independent of $\mathbf{q}$, hence a coupling strength parameter independent of $\mathbf{q}$.

The conservation of crystal momentum $\mathbf{q}+\mathbf{q}^{\prime}=\mathbf{q}^{\prime \prime}+k \mathbf{G}$ $(k=0,1)$ allows the $\mathbf{q}^{\prime \prime}$ of the diffuse modes to sweep over all the first Brillouin zone when $\mathbf{q}^{\prime}$ (TO modes) covers the first Brillouin zone, even for a single value of q. Finally, the anharmonicity in $\mathrm{Na}-\mathrm{Br}$ has no strong dependence on crystallographic direction and is dominated by first-neighbor interactions [25].

Consider first the case where $\mathrm{TA}+\mathrm{TO} \rightleftharpoons \mathrm{G}-\mathrm{LO}$ and the interacting TA, TO, and LO diffuse phonon modes can be treated as individual quantum oscillators with a coupling coefficient $\eta$. The total Hamiltonian is the same as Eq. (3). After dropping terms that do not conserve energy,

$$
\mathcal{H}_{\mathrm{sys}}=\mathcal{H}_{0}+\hbar \eta\left(\hat{a}_{j} \hat{a}_{j^{\prime}} \hat{a}_{j^{\prime \prime}}^{\dagger}+\hat{a}_{j}^{\dagger} \hat{a}_{j^{\prime}}^{\dagger} \hat{a}_{j^{\prime \prime}}\right)
$$

This is the same form as for parametric down-conversion in nonlinear optics. The mode coupling is enhanced resonantly when $\omega_{j^{\prime}}=\omega_{j^{\prime \prime}}-\omega_{j}$.

The second case TA $+\mathrm{TO} \rightleftharpoons \mathrm{G}$-TO has the same transverse polarization for two optical modes. These two TO modes can be modeled as a single oscillator. Its spectral weight is redistributed in energy owing to strong coupling to the TA mode. This is exactly the phonon intermodulation mechanism described with the quantum Langevin model.

The Supplemental Material [28] gives more details of how experimentally measured parameters of $\omega_{1} \simeq 16.97 \mathrm{meV}$, $\omega_{2} \simeq 8.2 \mathrm{meV}, \gamma_{1} \simeq 3.6 \mathrm{meV}$, and $\gamma_{2} \simeq 3.7 \mathrm{meV}$ were used in a numerical analysis that generated the spectral shapes of Fig. 1(b), with different coupling parameters $g$. In the weakcoupling case, our measured INS spectra would show no features other than the main peak at $\omega=\omega_{1}$. In the mediumcoupling case, the lower sideband peak at $\omega_{1}-\omega_{2}$ is only 
a shoulder on the main peak, but the upper sideband with $\omega_{1}+\omega_{2}$ should be distinct. The strong-coupling case shows two symmetric sidebands as shoulders on the main peak. The clear, isolated diffuse intensity "G" in Fig. 2(b) and weaker ILM are consistent with the TA and TO modes being in the medium-coupling case. To verify this, we solved numerically for the ratio between the heights of the two resonant peaks at $\omega=\omega_{1}$ and $\omega=\omega_{1}+\omega_{2}$ [Eq. (16)] to obtain the coupling strength parameter $|g| \simeq 3.7 \mathrm{meV}$, showing that the system is indeed in the medium-coupling domain. This also explains the difference in visibility between the ILM and the ghost phonon mode.

The prior treatment of ILMs [6] considered the dynamics of a classical system with linear and cubic terms in the restoring forces between neighboring atoms and showed the conditions for mode localization. Our approach with the quantum Langevin equation is better able to predict the spectral shape, providing deeper insights into the phonon intermodulation mechanism. Unlike a classical intermodulation, phonon intermodulation can have an asymmetric quantum effect of enhancing one sideband at the expense of the other.

Our recent paper on $\mathrm{NaBr}$ used perturbation theory to obtain thermal expansion, showing only modest disagreement with experiment at higher temperatures [25]. Thermodynamic effects of IPSs deserve more consideration, but the sidebands do not dominate the phonon spectrum, and the average of their energies is approximately the same as the average of the intermodulating phonons. Thermodynamic properties such as thermal expansion may not require precise assessments of sidebands.

Other materials with anharmonic phonons should have IPSs at modest temperatures. Different alkali halides are obvious candidates, as are materials with phonon instabilities, where nonlinear phonon interactions may generate sidebands as the instabilities grow. The sidebands in $\mathrm{NaBr}$ were from acoustic plus optic modes, but in principle, two anharmonic optic modes could also generate sidebands. Anharmonicity may offer a new functionality for optical materials in the infrared or a means to modulate visible light in ways that originate with phonon interactions, rather than an asymmetry in electronic polarizability. The ghost modes in $\mathrm{NaBr}$ decay rapidly into TA and TO modes through three-phonon processes. The two new phonons are in phase and would be entangled in the Einstein-Podolsky-Rosen sense. Their coherence time will be short, however.

\section{CONCLUSIONS}

A band of spectral intensity from high-energy phonons is predicted and observed in $\mathrm{NaBr}$. It is an IPS from anharmonic interactions between normal modes. Its partner, the lower sideband, is an ILM. The transfer of spectral weight to upper and lower IPSs likely occurs in other anharmonic materials, but the flat dispersions in $\mathrm{NaBr}$ make them easier to observe. The TO part of this feature is consistent with an IPS from the anharmonic coupling of TO modes and TA modes. The LO part is consistent with strong three-phonon process, again with anharmonic coupling to the TA modes. The spectral shapes and weights of the IPSs are altered by the quantum back action from the thermal bath. There are similarities to the formation of sidebands in laser-cavity experiments, which also depend on anharmonicity and quantum force fluctuations from the thermal bath. Compared with laser-cavity experiments with photons, the anharmonic sidebands in $\mathrm{NaBr}$ are a natural process that occurs in thermodynamic equilibrium, and both the interacting modes have the noise spectrum from the thermal bath. The IPS should be present at $0 \mathrm{~K}$ owing to couplings to the zero-point levels, and some traces may be visible in the dispersions at $10 \mathrm{~K}$. The spectral shapes of the two sidebands offer a probe of quantum noise, giving parameters for mode coupling and damping from the thermal bath. Perhaps the upper IPS could offer methods for the thermal control of light-matter interactions.

\section{ACKNOWLEDGMENTS}

We thank O. Hellman, K. Vahala, and F. Yang for helpful discussions. Research at the Spallation Neutron Source (SNS) and the High Flux Isotope Reactor (HFIR) at the Oak Ridge National Laboratory was sponsored by the Scientific User Facilities Division, Basic Energy Sciences (BES), Department of Energy (DOE). M.E.M. was supported by the US Department of Energy, Office of Science, Office of Basic Energy Sciences, Materials Sciences and Engineering Division under Contract No. DE-AC05-00OR22725. This paper used resources from National Energy Research Scientific Computing Center (NERSC), a DOE Office of Science User Facility supported by the Office of Science of the US Department of Energy under Contract No. DE-AC02-05CH11231. This paper was supported by the DOE Office of Science, BES, under Contract No. DE-FG02-03ER46055.
[1] P. M. Chaikin and T. C. Lubensky, Principles of Condensed Matter Physics (Cambridge University Press, Cambridge, 1995).

[2] J. M. Ziman, Electrons and Phonons: The Theory of Transport Phenomena in Solids (Oxford University Press, Oxford, 2001).

[3] A. A. Maradudin and A. E. Fein, Scattering of neutrons by an anharmonic crystal, Phys. Rev. 128, 2589 (1962).

[4] D. C. Wallace, Thermodynamics of Crystals (Wiley, New York, 1998).
[5] G. Grimvall, Thermophysical Properties of Materials (Elsevier, North-Holland, 1986).

[6] A. J. Sievers and S. Takeno, Intrinsic Localized Modes in Anharmonic Crystals, Phys. Rev. Lett. 61, 970 (1988).

[7] A. J. Sievers, M. Sato, J. B. Page, and T. Rössler, Thermally populated intrinsic localized modes in pure alkali halide crystals, Phys. Rev. B 88, 104305 (2013).

[8] M. Sato and A. Sievers, Direct observation of the discrete character of intrinsic localized modes in an antiferromagnet, Nature 432, 486 (2004). 
[9] M. E. Manley, A. J. Sievers, J. W. Lynn, S. A. Kiselev, N. I. Agladze, Y. Chen, A. Llobet, and A. Alatas, Intrinsic localized modes observed in the high-temperature vibrational spectrum of NaI, Phys. Rev. B 79, 134304 (2009).

[10] M. E. Manley, D. L. Abernathy, N. Agladze, and A. J. Sievers, Symmetry-breaking dynamical pattern and localization observed in the equilibrium vibrational spectrum of NaI, Sci. Rep. 1, 4 (2011).

[11] M. E. Manley, J. W. Lynn, D. Abernathy, E. Specht, O. Delaire, A. Bishop, R. Sahul, and J. Budai, Phonon localization drives polar nanoregions in a relaxor ferroelectric, Nat. Commun. 5, 3683 (2014).

[12] M. E. Manley, O. Hellman, N. Shulumba, A. F. May, P. J. Stonaha, J. Lynn, V. O. Garlea, A. Alatas, R. P. Hermann, J. D. Budai, H. Wang, B. C. Sales, and A. J. Minnich, Intrinsic anharmonic localization in thermoelectric $\mathrm{PbSe}$, Nat. Commun. 10, 1928 (2019).

[13] M. Kempa, P. Ondrejkovic, P. Bourges, P. Marton, and J. Hlinka, Lattice dynamics of $\mathrm{NaI}$ studied by inelastic neutron scattering: absence of thermally induced discrete breathers, Phys. Rev. B 89, 054308 (2014).

[14] S. Pailhès, H. Euchner, V. M. Giordano, R. Debord, A. Assy, S. Gomès, A. Bosak, D. Machon, S. Paschen, and M. de Boissieu, Localization of Propagative Phonons in a Perfectly Crystalline Solid, Phys. Rev. Lett. 113, 025506 (2014).

[15] A. Rivière, S. Lepri, D. Colognesi, and F. Piazza, Wavelet imaging of transient energy localization in nonlinear systems at thermal equilibrium: the case study of $\mathrm{NaI}$ crystals at high temperature, Phys. Rev. B 99, 024307 (2019).

[16] A. A. Clerk, M. H. Devoret, S. M. Girvin, F. Marquardt, and R. J. Schoelkopf, Introduction to quantum noise, measurement, and amplification, Rev. Mod. Phys. 82, 1155 (2010).

[17] T. J. Kippenberg and K. J. Vahala, Cavity optomechanics: backaction at the mesoscale, Science 321, 1172 (2008).

[18] K. Vahala, M. Herrmann, S. Knünz, V. Batteiger, G. Saathoff, T. Hänsch, and T. Udem, A phonon laser, Nat. Phys. 5, 682 (2009).

[19] J. Chan, T. M. Alegre, A. H. Safavi-Naeini, J. T. Hill, A. Krause, S. Gröblacher, M. Aspelmeyer, and O. Painter, Laser cooling of a nanomechanical oscillator into its quantum ground state, Nature 478, 89 (2011).

[20] F. Benz, M. K. Schmidt, A. Dreismann, R. Chikkaraddy, Y. Zhang, A. Demetriadou, C. Carnegie, H. Ohadi, B. de Nijs, R. Esteban, J. Aizpurua, and J. J. Baumberg, Single-molecule optomechanics in "picocavities", Science 354, 726 (2016).

[21] R. Riedinger, S. Hong, R. A. Norte, J. A. Slater, J. Shang, A. G. Krause, V. Anant, M. Aspelmeyer, and S. Gröblacher, Non-classical correlations between single photons and phonons from a mechanical oscillator, Nature 530, 313 (2016).

[22] W. Renninger, P. Kharel, R. Behunin, and P. Rakich, Bulk crystalline optomechanics, Nat. Phys. 14, 601 (2018).

[23] A. Tavernarakis, A. Stavrinadis, A. Nowak, I. Tsioutsios, A. Bachtold, and P. Verlot, Optomechanics with a hybrid carbon nanotube resonator, Nat. Commun. 9, 662 (2018).

[24] D. L. Abernathy, M. B. Stone, M. Loguillo, M. Lucas, O. Delaire, X. Tang, J. Lin, and B. Fultz, Design and operation of the wide angular-range chopper spectrometer arcs at the spallation neutron source, Rev. Sci. Instrum. 83, 015114 (2012).

[25] Y. Shen, C. N. Saunders, C. M. Bernal, D. L. Abernathy, M. E. Manley, and B. Fultz, Anharmonic Origin of the Giant Thermal Expansion of NaBr, Phys. Rev. Lett. 125, 085504 (2020).
[26] The internal structure of the ARCS spectrometer has radial, platelike baffles of neutron absorbing material that block stray scattering off detector tubes from reaching other detectors. This works well, except for detectors located approximately $180^{\circ}$ across the detector array. Those neutrons, elastically scattering across the diameter of the instrument, arrive at a later time than the main elastic peak, appearing to be at an inelastic energy transfer of $80 \%$ of $E_{i}$. In the prior dataset of $E_{i}=30 \mathrm{meV}$, this artifact appears at $24 \mathrm{meV}$, overlapping the ghost modes. For the present dataset with $E_{i}=50 \mathrm{meV}$, its $40 \mathrm{meV}$ artifact is safely out of range, although the energy resolution of the instrument is approximately $\frac{5}{3}$ times larger than the prior dataset.

[27] D. S. Kim, O. Hellman, J. Herriman, H. L. Smith, J. Y. Y. Lin, N. Shulumba, J. L. Niedziela, C. W. Li, D. L. Abernathy, and B. Fultz, Nuclear quantum effect with pure anharmonicity and the anomalous thermal expansion of silicon, Proc. Natl. Acad. Sci. USA 115, 1992 (2018).

[28] See Supplemental Material at http://link.aps.org/supplemental/ 10.1103/PhysRevB.103.134302 for analysis of the quantum Langevin equation, corrections for experimental background, and the temperature dependence of the IPS.

[29] G. Kresse and J. Hafner, Ab initio molecular dynamics for liquid metals, Phys. Rev. B 47, 558 (1993).

[30] G. Kresse and J. Hafner, Ab initio molecular-dynamics simulation of the liquid-metal-amorphous-semiconductor transition in germanium, Phys. Rev. B 49, 14251 (1994).

[31] G. Kresse and J. Furthmüller, Efficiency of ab-initio total energy calculations for metals and semiconductors using a plane-wave basis set, Comput. Mater. Sci. 6, 15 (1996).

[32] G. Kresse and J. Furthmüller, Efficient iterative schemes for $a b$ initio total-energy calculations using a plane-wave basis set, Phys. Rev. B 54, 11169 (1996).

[33] G. Kresse and D. Joubert, From ultrasoft pseudopotentials to the projector augmented-wave method, Phys. Rev. B 59, 1758 (1999).

[34] J. P. Perdew, K. Burke, and M. Ernzerhof, Generalized Gradient Approximation Made Simple, Phys. Rev. Lett. 77, 3865 (1996).

[35] M. Gajdoš, K. Hummer, G. Kresse, J. Furthmüller, and F. Bechstedt, Linear optical properties in the projectoraugmented wave methodology, Phys. Rev. B 73, 045112 (2006).

[36] X. Gonze and C. Lee, Dynamical matrices, born effective charges, dielectric permittivity tensors, and interatomic force constants from density-functional perturbation theory, Phys. Rev. B 55, 10355 (1997).

[37] A. Togo and I. Tanaka, First principles phonon calculations in materials science, Scr. Mater. 108, 1 (2015).

[38] O. Hellman, I. A. Abrikosov, and S. I. Simak, Lattice dynamics of anharmonic solids from first principles, Phys. Rev. B 84, 180301 (2011).

[39] O. Hellman, P. Steneteg, I. A. Abrikosov, and S. I. Simak, Temperature dependent effective potential method for accurate free energy calculations of solids, Phys. Rev. B 87, 104111 (2013).

[40] O. Hellman and I. A. Abrikosov, Temperature-dependent effective third-order interatomic force constants from first principles, Phys. Rev. B 88, 144301 (2013).

[41] C. W. Gardiner and M. J. Collett, Input and output in damped quantum systems: quantum stochastic differential 
equations and the master equation, Phys. Rev. A 31, 3761 (1985).

[42] T. E. Markland and M. Ceriotti, Nuclear quantum effects enter the mainstream, Nat. Rev. Chem. 2, 0109 (2018).

[43] The HB3 spectrometer was operated to maintain a constant $\vec{Q}$ for $300 \mathrm{~K}$, without correcting for thermal expansion, which shrinks the Brillouin zones of the crystal. The measured $\vec{Q}$ therefore increases as the crystal expands, and the shift of the TA mode in Fig. 4 is consistent with the slope of the TA dispersion.

[44] N. Shulumba, O. Hellman, and A. J. Minnich, Intrinsic localized mode and low thermal conductivity of PbSe, Phys. Rev. B 95, 014302 (2017). 